\title{
The Importance of the Verbal Written Handover Report in Hospital
}

\author{
Iqra Shafi ${ }^{1 *}$, Sana Sehar ${ }^{2}$, Muhammad Afzal $^{3}$, Dr. Syed Amir Gilani ${ }^{4}$ \\ ${ }^{1}$ BSN(Student),Lahore School of Nursing, The university of Lahore 1 Km Rairwind Rd, Sultan Town, Lahore, Punjab, Pakistan \\ ${ }^{2}$ Assistant professor at LSN(UOL), Lahore School of Nursing , The university of Lahore 1 KmRairwind Rd, Sultan Town, Lahore, Punjab, Pakistan \\ ${ }^{3}$ Professor at LSN(UOL), Lahore School of Nursing, The university of Lahore 1 KmRairwind Rd ,Sultan Town, Lahore, Punjab , Pakistan \\ ${ }^{4}$ Dean Faculty of Allied Health Sciences, Lahore School of Nursing ,The university of Lahore 1 Km Rairwind Rd ,Sultan Town, Lahore, Punjab, \\ Pakistan
}

\author{
DOI: $10.36348 /$ sjnhc.2019.v02i12.005 \\ | Received: 05.12.2019 | Accepted: 15.12.2019 | Published: 26.12.2019 \\ *Corresponding author: Iqra Shafi
}

\section{Abstract}

Provides reliable contact information about patient care and safety. Analysis of the lack of reports between hospital and emergency room personnel indicates incomplete or lack of general information. Nurses play an important role in dissemination and dissemination of information by using nurse directives. Sharing information in a hospital affects patient safety and treatment plans and delivery based on this information. Test modifications that were used for the comfort of patients entering the ambulance for several days decided to participate in this study. Sample size should take into account sales that occur evenly throughout the month, with each change and day of the week. Documenting the content of the reports submitted will affect breastfeeding practices. You can also use the standard login format and technology to improve content delivery.

Keywords: Verbal Written, reliable contact, Nurses.

\begin{abstract}
Copyright @ 2019: This is an open-access article distributed under the terms of the Creative Commons Attribution license which permits unrestricted use, distribution, and reproduction in any medium for non-commercial use (NonCommercial, or CC-BY-NC) provided the original author and source are credited.
\end{abstract}

\section{INTRODUCTION}

Handover can be portrayed as a procedure that includes the passing and acknowledgment of obligation regarding a few or all parts of care for a patient, or gathering of patients, and the sharing of important data. Move handovers are a customary element of human services work, occurring among approaching and active staff when there is a move change. Move handover is a key device in guaranteeing instructive congruity, which thus is basic for progression of care [1].

Move handovers are getting increasingly visit, because of shorter. Working hours for specialist's attendants and paramedical staff an aftereffect of guidelines, for example, the European Working Time Directive. Be that as it may, various examinations feature the job of move handovers in unfavorable occasions. This, joined with the expanded recurrence of move handovers, has prompted enthusiasm for giving mechanical help to handover[2].

Handovers additionally react to the nearby setting, with what considers vital or fundamental data to hand over shifting as indicated by the therapeutic forte, the clinicians' assurance about the patient's condition, the seriousness and stability of the patient's condition, and the outstanding task at hand of staff individuals[3].

Handovers ought to likewise be viewed as arranged inside a specific spatial condition which can possibly affect the correspondence. For instance, handovers may occur in a room away from the ward or may happen in a moren'public space, for example, by the bedside. One examination proposes that ma bedside handover enables approaching staff to present questions that may not emerge away from the patient.

The information provided and the nature of the communication also depends on who is involved in the handover. For example, the amount of information handed over may depend on whether or not an oncoming member of staff has previously cared for the patient .Also important is the participants' place within the professional hierarchy and their level of experience and responsibility. For example, junior staff nurse and doctors have been found to have a narrow definition of handover, focusing on tasks to be completed by the end of the shift [4].

While verbal face to face handovers predominate, and have been found to be preferred by clinicians, these do not always occur due to, for 
example, time constraints and patients being widely dispersed. Some studies emphasis the conversational nature of handovers, with a two-way exchange of information between outgoing and oncoming staff. However, other studies of both medical and nursing handovers have found questions being asked of the person giving the handover occur infrequently, suggesting that, in those particular contexts, handover was more of a report and less of a conversation.

\section{BACKGROUND CASE}

Case site is a 46-bed general emergency ward in a public hospital.The majority of patients on this ward is elderly and many require palliative care. It is a short-stay ward where patients are assessed and either discharged from hospital or transferred to an appropriate ward. Due to the nature of the ward, patients of a wide range of ages and with a broad range of conditions are seen. The ward takes both elective and emergency angioplasty patients. Patients are transferred from the ward and then transferred back to the ward following their operations and other consultation.

Patients who deserved one-day treatment were recruited for daytime surgery. Patients were admitted to the angiography department for an antibody generally 6 inches. Heparin (3000 units) was given at the beginning of the procedure. At the end of the procedure, the femoral sheath was removed and the radiologist pressed the position of the inguinal puncture until the homeostasis disappeared. Arterial occlusion devices were not recurrently used.

When the patient is shifted to the ward after angiography and angioplasty, neither the staff nurse, doctor nor the worker never tell about what happen to patient. Nor is the patient advised that you do not move her leg for two hours after angio plasticity. Most of the time after angiography femoral and radial sheath out after procedure. But the sheath packs for two or three hours after angioplasty. Once upon a time, a patient had a sheet pack after angio plasticity and worker left the patient in ward in such a condition that he did not give any one over about patent, and patient did not know precautionary measures about angioplasty. Patient received from morning shift with incomplete handover .procedure was not informed to next shift. After half an hour as the patient sit bleeding from femoral site. Patient collapse suddenly with low blood pressure and pulse. Immediately call to on duty doctor and dresser and shift the patient in emergency ICU. The number of these incidents was increasing in hospital due lack of communication between staff nurses and pare medical staff. Action was taken by nursing superintendent against this negligence.

\section{DISCUSSION}

Post-procedure care, including puncture site inspection and monitoring of vital signs was undertaken by nursing staff in the department. The ankle brachial pressure index was measured using hand-held Doppler by a vascular laboratory technician. Patients were then transferred to the day surgery ward accompanied by a trained nurse. Supine bed rest was maintained for $3 \mathrm{~h}$ after which time the patients were mobilized progressively and, assuming no puncture site or other problems, were discharged by a radiology nurse $5 \mathrm{~h}$ after completion of the procedure [5].

We have provided a description of medical and nursing shift handovers across three varied case sites. The description of the general features of the handovers in each case site highlights some of the challenges of having a face to face handover. Shift handovers often start late, not all staff may be present for all of the handover, and interruptions are likely. This is due to a contradiction inherent in handover that has long been acknowledged; that, while trying to ensure continuity of care, handover often results in a disruption of care as members of staff leave their duties [6].

The results from this study support the use of electronic handover to improve efficiency of patient information transferred between doctors. Furthermore, it supports the continued use and development of this electronic format for handover particularly as it serves as a user-friendly, low-cost, efficient means of transfer of patient details among staff post-call leading to better continuity of care for patients. Importantly, this is the first study to show that use of electronic handover is associated with a significant reduction in patient length of stay. This is despite the pilot nature of this investigation [7].

There is a danger that new devices are introduced into clinical practice in an uncritical way, such that their use becomes the accepted standard of practice with inevitable consequences in terms of cost and complexity of the procedure. The main driver for the use of closure devices is the presumption that patients can mobilize sooner. The duration of bed-rest prescribed after manual compression has gradually been reduced over recent years. However, a safe minimum duration of bed-rest has never been established. All research into bed-rest has favoured the shorter duration over the longer [2].

The goal of this study was to describe and quantify a critical aspect of inter hospital handoffs that impacts patient outcomes. We developed a novel tool to evaluate transfer documentation quality. When controlling for severity of illness, the completeness of documentation was associated with lower rates of inhospital mortality, adverse events, and labor duplication. Complete objective information, such as laboratory results and imaging, seemed to disproportionately improve outcomes [8].

Identified a need for improvement in the handoff report contents and communication between 
pre-hospital and E.D. staff. There is also correlation to the evidence that there is need for a standardization of a handoff reporting tool that could be utilized by the Prehospital care providers which could potentially improve the information transfer and impact patient safety. There is a need for input into this design by providers and nursing staff who use the tool to give handoff report. There should also be input from staff into future changes to the electronic documentation system, which could impact overall compliance with documentation Requirements [9].

\section{Problem focused}

Just as being for all intents and purposes centered, the handover reports could likewise be depicted as issue centered. For instance, on the off chance that site crisis ward nurture commonly didn't state the patient's conclusion or past therapeutic history. Rather, the accentuation was on changes to the patient's consideration (for example drugs that are never again being given), parts of the patient express that were worrisome, parts of the patient express that they were observing), parts of the patient's consideration needs that were deviations from the standard or dangerous (for example in the event that the patient was uniquely to have pureed nourishment), occasions that occurred during the past move (falls, heaving, conduct, for example, yelling), and, as effectively portrayed, errands to be finished. This attention on issues implied that there could be noteworthy variety in the measure of data given about specific patients, as appeared in this field note remove from an early daytime nursing shift handover [10].

\section{PROBLEM SOLVING PROCESS} the issue:

We apply problem solving process to resolve

\section{Define the problem}

The nursing change of shift report or handover is a communication that occurs between two shifts of nurses whereby the specific purpose is to communicate information about patients under the care of nurses [11].

\section{Gather information}

hospital.

Information was collected from a public

\section{Analyze the information \\ Analyze information to other general hospitals information which was different.}

\section{Develop solution}

The number of these incidents was increasing in hospital due lack of communication between staff nurses and pare medical staff.

\section{Make a decision}

We make a decision to take action against negligence of patient.

\section{Implement the decision}

For the implementation of a decision first we inform Nursing Superintendent, and Chief Executive that the multiplies time patients was neglected due to lack of communication between nurses, doctors and Para-medical staff. Separate beds should be arranged for the patients with angiography and angioplasty including specialized staff.

\section{Evaluate the solution}

The solution which was given by us is carried out properly and gave positive results.

\section{MANAGEMENT PROCESS}

Improvement of the complex, including identification of the site and maintenance of vital signs performed by nursing staff within the department. Square root of the nail was measured using Doppler and radius. Patients will be given a working day with a trained nurse. The bed was left for 3 hours, and then the patient was repaired slowly, assuming that any complication or other complication was not resolved with radiotherapy 5 after discharge. During discharge, the patient was given a telephone number from the Vascular Radiology department (to be used during the procedure) and a telephone call to another registered nurse (for use outside the clock) used in case of problems or problems. If the person thinks it is inappropriate to give up, the manager next to the affected bed will allow for a good night's sleep. At night these beds are not shared in advance [4].

Reporting in hospitals and emergency rooms can affect patient safety, which can be improved by potential changes to the referral reporting workflow and the use of standard reporting tools. In the future, there should be projects and studies aimed at developing electronic documentation and documentation tools based on medical records corresponding to this tool. The project has shown that it does not comply with the electronic documentation of the transfer report and that the exchange of information during the report is limited. The role and performance of electronic systems for performance and efficiency that affect patient care in E.D. In the field of nursing, there are opportunities to advance nursing education that are important for the quality of education and how they affect patient care and safety. Psychological research is important, as are all outcomes due to inadequate exposure. It is also important to examine the consequences of complaints and the benefits of breastfeeding [11]. 


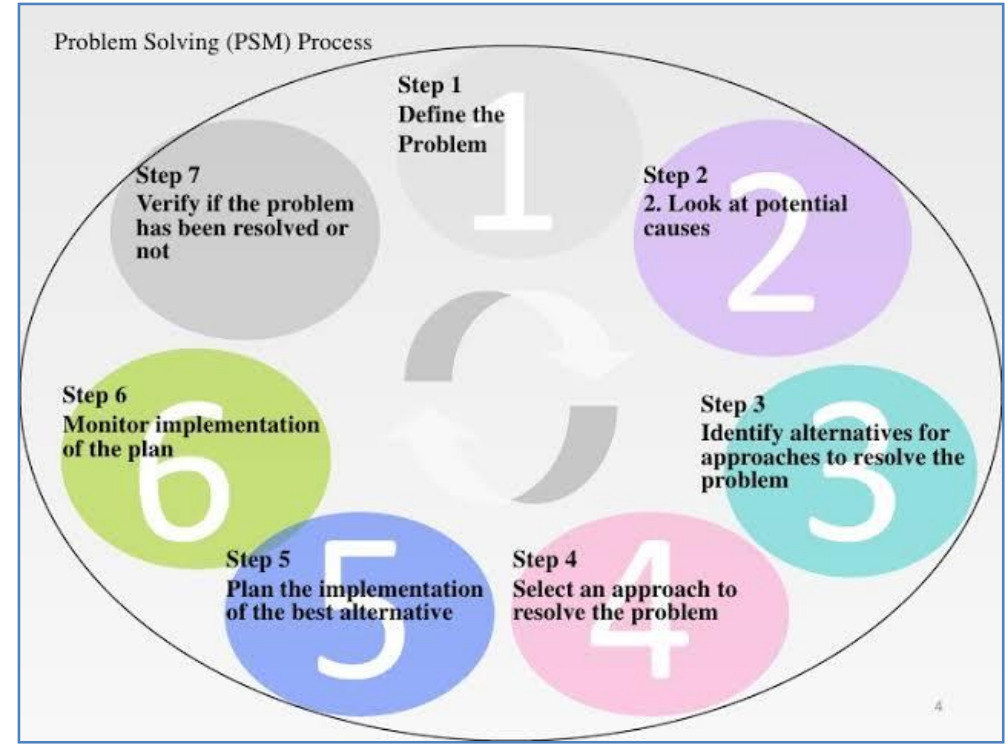

\section{CONCLUSION}

Bed side nurses raises awareness of the nurse's role in communicating patient safety and satisfaction. The public expects greater transparency in the care of health care patients, only for patients with adequate care plans. Changes in the communication of nurses, therapists, patients / families and members of the health team include safety and well-being [11].

\section{REFERENCE}

1. Randell, R., Wilson, S., \& Woodward, P. (2011). The importance of the verbal shift handover report: a multi-site case study. International journal of medical informatics, 80(11), 803-812.

2. Wears, R. L., Hollnagel, E., \& Braithwaite, J. (Eds.). (2015). Resilient health care, volume 2: the resilience of everyday clinical work. Ashgate Publishing, Ltd.

3. Kasthuri, R., Karunaratne, D., Andrew, H., Sumner, J., \& Chalmers, N. (2007). Day-case peripheral angioplasty using nurse-led admission, discharge, and follow-up procedures: arterial closure devices are not necessary. Clinical radiology, 62(12), 1202-1205.

4. Espiritu, M., Patil, U., Cruz, H., Gupta, A., Matterson, H., Kim, Y., \& Mally, P. (2014).
Evacuation of a neonatal intensive care unit in a disaster: lessons from Hurricane Sandy. Pediatrics, 134(6), e1662-e1669.

5. Gramling, S. G. (2018). A Mixed Methods Study of The Symptom Experience Following Endovascular Treatment for Lower Extremity Peripheral Arterial Disease (Doctoral dissertation, Mercer University).

6. Fealy, G. (2016). "Clinical handover practices in maternity services in Ireland: A qualitative descriptive study." Midwifery, 39: 20-26.

7. Ryan, S. (2011). "Impact of a new electronic handover system in surgery." International Journal of Surgery, 9(3): 217-220.

8. Usher, M. G. (2016). "Information handoff and outcomes of critically ill patients transferred between hospitals." Journal of critical care 36: 240245.

9. Matichko, K. (2015). Handoff Communication in the Emergency Department. DNP Forum.

10. Bowen, S. (2015). "The impact of language barriers on patient safety and quality of care." Ottawa, ON, Canada: Société Santé en français.

11. Bost, N. (2010). "Clinical handover of patients arriving by ambulance to the emergency department-a literature review." International emergency nursing, 18(4): 210-220. 